\title{
Detection and Classification of Human Arm Movements for Physical Rehabilitation
}

\author{
A.R. Guraliuc ${ }^{(1)}$, A.A. Serra ${ }^{(1)}$, P. Nepa* ${ }^{(1)}$, \\ G. Manara ${ }^{(1)}$, F. Potorti ${ }^{(2)}$
}

(1) Dept. of Information Engineering, University of Pisa, Pisa, Italy

(2) ISTI, CNR, Pisa, Italy

E-mail: anda.guraliuc, andrea.serra, p.nepa, g.manara@iet.unipi.it;

potorti@isti.cnr.it

\section{Introduction}

In the latest years, the detection of body posture and activity received a significant interest in the field of the physical rehabilitation aimed at providing advanced medical therapies to patients who have suffered a stroke, joint replacements/reconstructions, amputation, or some motor function disability resulting from Parkinson's disease [1,2]. Rehabilitation is a dynamic process and the restoration of patients' functional capability to normal requires every day functional activities that need to be monitored and controlled by specialized medical operators. An effective approach is represented by motion capturing systems where some video cameras follow the movements of a number of markers placed on the human body to reconstruct its activity. However, such systems are complex, expensive and require a large number of constitutive elements (cameras and markers) [3].

Lately, great improvements derived from the progress of recent wearable wireless sensors networks that could replace the existing wired telemetry systems [4]. In this case, the patient recovery and rehabilitation can be remotely supervised and subsequently corrected. In a typical scenario, a patient wears up some sensors forming an on-body sensor network, then a base station registers the data collected from it. Afterward, processing analysis through a home computer or a laptop can be performed on the registered data to detect, evaluate and measure the effect of patient rehabilitation movements. Continuous monitoring will allow the patients to have a normal life reducing the inconvenience of regular visits to the physician. Wearable Wireless Body Area Networks (WBANs) can yield quantitative data that can be used to better understand the disease and develop more effective treatments. Furthermore they can provide a less expensive rehabilitation healthcare.

In this paper an arm rehabilitation scenario was set-up to estimate and evaluate the principal activities of this limb by the suggested WBAN-based approach. A couple of wearable sensors mounted on the arm and a fixed node constitute the wireless network. In order to identify and classify the rehabilitation activities an algorithm based on the Received Signal Strength Indicator (RSSI), a parameter already available in the wireless sensor nodes, was applied. As a first attempt, a support vector machine (SVM) learning technique was implemented. 


\section{System description, methodology and experimental results}

In this study, the WBAN was formed by the IRIS transceiver modules from Crossbow $^{\mathrm{TM}}$ [5], which operate at $2.4 \mathrm{GHz}$ according to the IEEE 802.15.4 protocol. They are placed on the arm and at a fixed node connected to a PC. Measurements were performed in an office environment and the transmission power level was set to $3 \mathrm{dBm}$. The IRIS motes form an ad-hoc on-body sensor network with a tree topology determined by the relative location on the body and by the wireless link characteristics. The network consisted of three types of sensors as shown in Figure 1. The gateway was the root of the entire topology and was connected directly to the processing server. The TX node was placed at the centre of the front waist. Finally, the RXs (namely IRIS27\&28) are placed on the right arm, one at the wrist and one on the upper part of the arm. In our experiments, each sensor was fixed with an elastic band so that the sensors do not change the position during the experiment. The TX periodically sends a beacon packet with a fixed transmission power. The RXs sample the received beacon, calculate the RSSI and send it back to the gateway node. The sampling data rate was $8 \mathrm{~Hz}$.

Some typical kinesitherapy practical exercises were considered as shown in Figure 2. By referring to arm rehabilitation, the exercises consist in: "Activity 1", where the arm was oscillating straight ahead from down to half; "Activity 2", where the arm was oscillating on side from down to half; and "Activity 3", which consists in the forearm movement. Each activity was performed for 30 seconds and repeated fifty times, and the RSSI was registered. Figure 3 presents some RSSI registration samples for the above activities. By applying a Discrete Fourier Transform (DFT) we are able to identify the activity velocity through the detection of its first-harmonic frequency. An example is shown in Figure 4. First, for a slow rate of the "Activity 1 " a frequency of $0.4 \mathrm{~Hz}$ was obtained. Then, for a faster rate the frequency increases to $1.2 \mathrm{~Hz}$. Above values are directly related to the activity rate ( 24 and 72 oscillations per minute, respectively).

A post registration elaboration data was performed in order to identify and classify the arm activities on the base of some RSSI statistical features: mean value, standard deviation and cross-correlation; the peak value of the first harmonic of the Discrete Fourier Transform has been considered as well.

A learning technique known as Support Vector Machine (SVM) was chosen due to the success of this approach in many classification problems [6-8]. This technique involves a training and a testing phase, which contain some data instances. Each instance in the training set contains one "target value" (class labels) and several "attributes" (features). The goal of SVM is to produce a model which predicts target value of data instances in the testing set in which only the features are given. The specific SVM implementation was based on the OneAgainst-One approach to tackle the multiclass classification problem. The classification is done by a max-wins voting strategy. A specific classifier is trained for every pair of classes (in our case one class is one activity). For a test sequence, each classifier assigns one vote with regard to the data set class labels, and the class with the maximum number of votes is the label assigned to the test sample. 
Each class was characterized through seven different features derived from RSSI data: the mean value and the standard deviation for both RXs (IRIS27 and IRIS28), the cross-correlation, and the peak value of the first harmonic of the Discrete Fourier Transform (for both RXs). A tenfold number was used for the training vector for each activity. A tenfold cross-validation technique was used. A sequence of the above three activities was used to test the algorithm accuracy, and a good match was noticed. Specifically, an $80 \%$ matching probability was obtained over a total of 150 measurements.

\section{Conclusions}

In this paper an experimental framework for arm rehabilitation movements detection was presented. Some typical rehabilitation exercises were performed with low cost IRIS motes used for on-body wireless sensor networks. An SVM learning technique was used to determine and classify the arm movements. Preliminary tests demonstrated that the method can deliver good detection performances. Work is in progress for leg rehabilitation exercises, as well as more complex situations. More results will be shown at the conference.

\section{Acknowledgements}

The authors wish to thank Sara Marongiu for her precious help in the measurement campaign.

\section{References}

[1] H. Zhou, H. Hu, "Human motion tracking for rehabilitation-A survey", Biomedical Signal Processing and Control, vol. 3, no. 1, p. 1-18, January 2008.

[2] S. Patel, K. Lorincz, R. Hughes, N. Huggins, J. Growdon, D. Standaert, M. Akay, J. Dy, M. welsh, P. Bonato, "Monitoring motor fluctuations in patients with Parkinson's disease using wearable sensors", IEEE Trans. On Information Technology in Biomedicine, vol. 13, no. 6, November 2009.

[3] M. Galli, C. Rigoldi, L. Mainardi, N. Tenore, P. Onorati, G. Albertini, "Postural control in patients with Down syndrome", Disability \& Rehabilitation, vol. 30, no. 30 (17), p. 1274-1278, 2008.

[4] K. Lorincz, B. Chen, G. W. Challen, A. R. Chowdhury, S. Patel, P. Bonato, M. Welsh, "Mercury: A wearable sensor network platform for high-fidelity motion analysis", Embedded Networked Sensor Systems Conf.(SenSys'09), 4-6 November 2009.

[5] http://www.xbow.com

[6] F. Naya, R. Ohmura, F. Takayanagi, H. Noma, K. Kogure, "Workers' routine activity recognition using body movements and location information", IEEE International Symposium on Wearable Computers, p.105-108, October 2006.

[7] P. Shenoy, K. J. Miller, J. G. Ojemann, R. P. N. Rao, "Finger movement classification for an electrocorticograohic BCI", IEEE EMBS Conf. on Neural Engineering, 2-5 May 2007.

[8] H. Y. Lau, K. Y. Tong, H. Zhu, "Support vector machine for classification of walking condition using miniature kinematic sensors", Med. Biol. Eng. Comput., vol. 46, no. 6, p. 563-573, June 2008. 


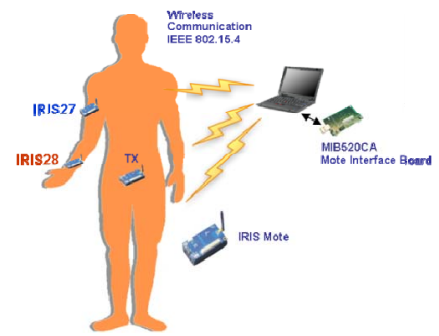

Fig. 1. Wearable wireless sensor network.
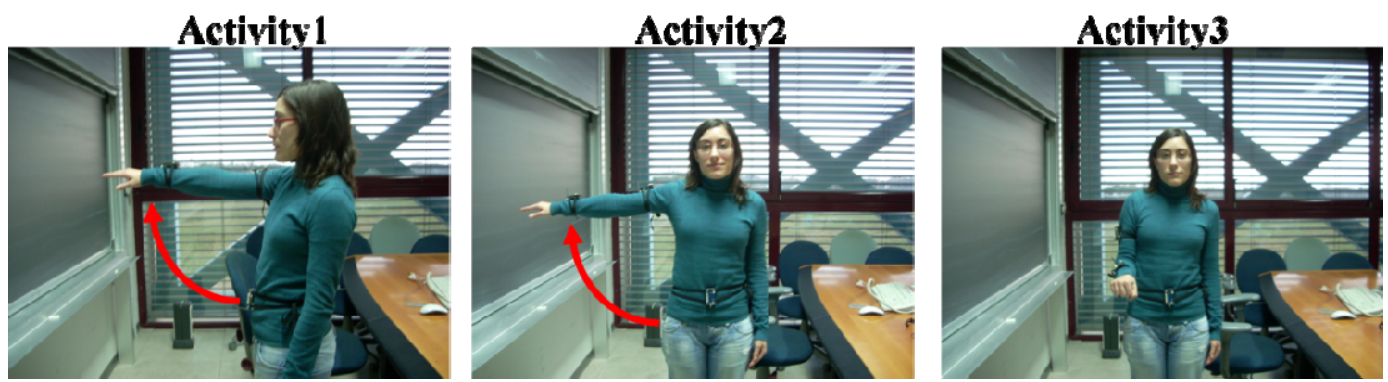

Fig. 2. Typical exercises for arm rehabilitation performed by the subject during the experiment: Activity1- arm oscillating straight ahead from down to half; Activity2- arm oscillating on side from down to half; Activity3- forearm movement.
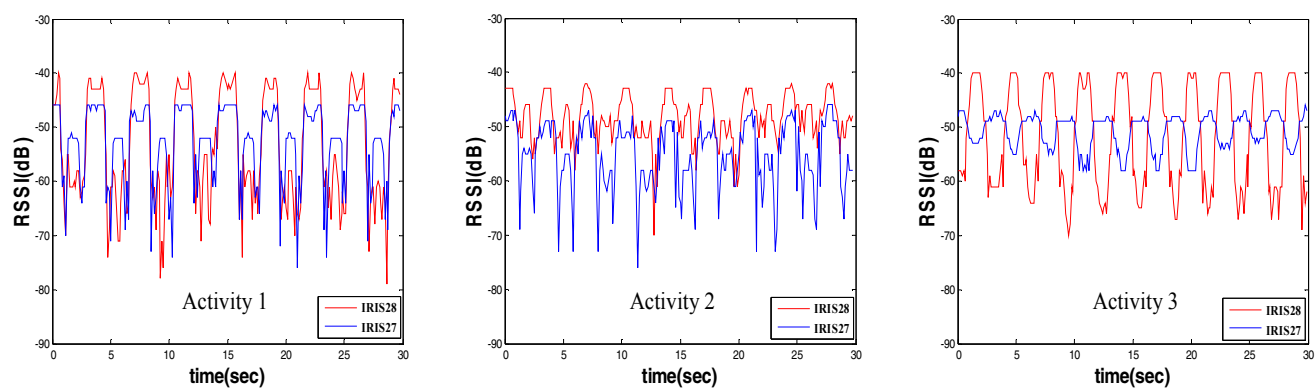

Fig. 3. RSSI readings for Activity1, Activity2 and Activity3.
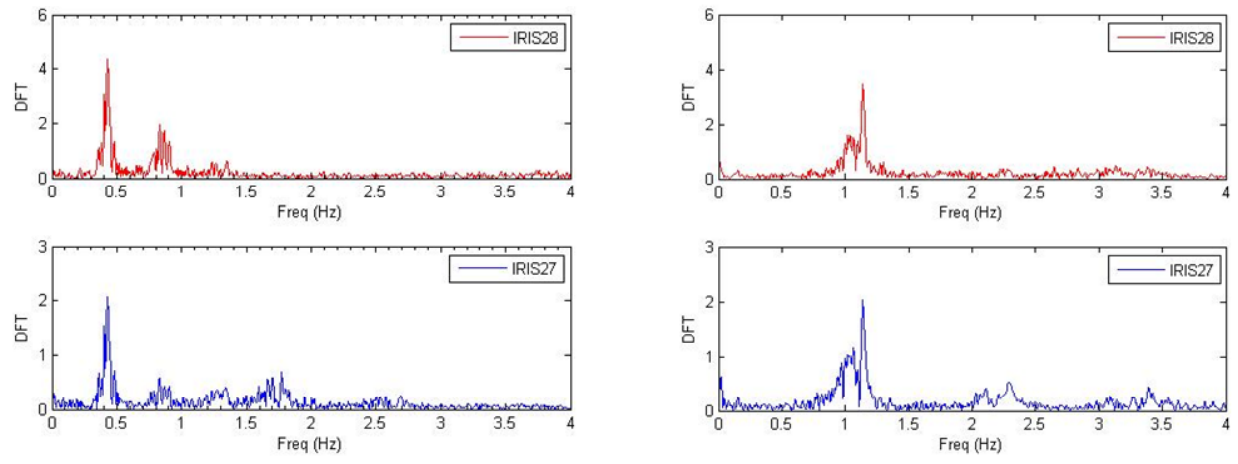

a).

b).

Fig. 4. Discrete Fourier Transform for Activity 1: a) slow activity, b) fast activity. 